\title{
Expression and function of transforming growth factor- $\beta$-activated protein kinase 1 in gastric cancer
}

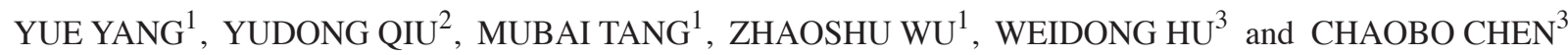

\author{
${ }^{1}$ Department of Surgery, The Third Affiliated Hospital, Nanjing University of Traditional Chinese Medicine, Nanjing, \\ Jiangsu 210001; ${ }^{2}$ Department of Hepatopancreatobiliary Surgery, Drum Tower Hospital, Medical School of Nanjing University, \\ Nanjing, Jiangsu 210000; ${ }^{3}$ Department of General Surgery, Wuxi Xishan People's Hospital, Wuxi, Jiangsu 214011, P.R. China
}

Received June 20, 2016; Accepted May 2, 2017

DOI: $10.3892 / \mathrm{mmr} .2017 .6998$

\begin{abstract}
The present study aimed to investigate the expression and role of transforming growth factor (TGF) - $\beta$-activated protein kinase 1 (TAK1) in human gastric cancer. Immunohistochemistry was performed to investigate the expression of TAK1 in surgical specimens of human gastric cancer tissue and adjacent normal tissue. The association between TAK1 and clinicopathologic factors was analyzed and the association between TAK1 expression and the overall survival rates was evaluated using Kaplan-Meier curves. In addition, the effect of the TAK1 selective inhibitor 5Z-7-oxozeaenol (OZ) on the biological characteristics of MGC803 human gastric cancer cells in vitro were investigated. The role of TAK1 in gastric cancer cell proliferation, apoptosis and invasion were determined by cell proliferation assays, flow cytometry analysis and transwell invasion assays, respectively. The findings of the present study demonstrated that the positive expression rate of TAK1 in gastric cancer and adjacent normal tissues was 70.5 and $25.9 \%$, respectively. Furthermore, TAK1 expression was significantly associated with advanced $\mathrm{N}$ stage and pathological stage $(\mathrm{P}<0.05)$. Survival analysis of 139 patients with gastric cancer indicated a lower overall survival rate of patients in the TAK1-positive group compared with the TAK1-negative group $(\mathrm{P}<0.05)$. In addition, treatment with the TAK1 selective inhibitor OZ reduced the proliferation and invasion abilities of MGC803 cells and significantly reduced the expression levels of phosphorylated-TAK1 (Thr187), nuclear p65, cyclin D1, Bcl-2 apoptosis regulator and matrix metallopeptidase (MMP)9 $(\mathrm{P}<0.05)$. OZ treatment significantly increased the expression levels of cytosolic cytochrome $c$ and cleaved caspase 3 and the apoptosis rate in MGC803 cells $(\mathrm{P}<0.05)$. In conclusion, these findings suggest
\end{abstract}

Correspondence to: Dr Chaobo Chen, Department of General Surgery, Wuxi Xishan People's Hospital, 588 Guangrui Road, Wuxi, Jiangsu 214011, P.R. China

E-mail: chaobochenwuxi@163.com

Key words: gastric cancer, transforming growth factor- $\beta$-activated kinase 1,5Z-7-oxozeaenol that increased TAK1 expression may be involved in the progression of gastric cancer; therefore, TAK1 may be used as a future therapeutic target for gastric cancer treatment.

\section{Introduction}

Gastric cancer has one of the highest cancer-associated mortalities worldwide and patients have a particularly high susceptibility to lymph node metastasis $(1,2)$. The incidence of gastric carcinoma has recently increased, which may be due to various environmental and social factors (3), including $H$. pylori infection, low socioeconomic status and perhaps dietary factors such as low consumption of fruits and vegetables and a high intake of salty and smoked food (4). Although higher overall survival rates for patients with gastric carcinoma are currently observed due to improved early cancer detection and increased use of radical surgery, gastric carcinoma remains the fourth most common cancer and is considered to be the second major cause of cancer-associated deaths globally (5). It is of note that effective methods for early diagnosis, monitoring for metastasis and prognosis are remain to be established for gastric cancer (6). Therefore, the identification of novel therapeutic targets for gastric cancer is required.

Transforming growth factor- $\beta$-activated kinase 1 (TAK1) regulates the nuclear factor- $\kappa \mathrm{B}(\mathrm{NF}-\kappa \mathrm{B})$ and mitogen-activated protein kinase (MAPK) signaling pathways, which have important roles in various biological processes, including development, cell survival, immune responses, metabolism and carcinogenesis (7). Previous studies demonstrated that TAK1 functions as a tumor promoter in various tissues, including breast and thyroid cancer $(8,9)$. TAK1 inhibition has also been reported to induce cancer cell death $(10,11)$, indicating that targeting TAK1 may be useful in the development of treatments for gastric cancer. Therefore, in order to develop cancer therapies that target TAK1, it is important to determine the regulation and role of TAK1 in the pathogenesis (12). To the best of our knowledge, the role of TAK1 in gastric cancer has not previously been investigated. 5Z-7-Oxozeaenol, a natural product of fungal origin, was reported to be a TAK1 specific inhibitor (13). Therefore, the present study investigated the expression of TAK1 in gastric cancer and its clinical significance, and further investigated the function of TAK1 in the development and progression of gastric cancer in vitro 
using 5Z-7-oxozeaenol (OZ), which is a selective TAK1 inhibitor (14).

\section{Materials and methods}

Patients. Gastric cancer samples and adjacent normal tissue samples used in the present study were obtained from 139 patients with gastric cancer that underwent resection at The Third Affiliated Hospital, Nanjing University of Traditional Chinese Medicine (Nanjing, China) and Wuxi XiShan People's Hospital (Wuxi, China) between January 2005 and August 2010. Normal gastric mucosa tissue $(\leq 5 \mathrm{~cm})$ adjacent to the tumor was excised and confirmed to be tumor-free following pathological analysis. Every resection specimen was examined by the Department of Pathology, The Third Affiliated Hospital, Nanjing University of Traditional Chinese Medicine (Nanjing, China), to confirm their histology features. The patients consisted of 81 males and 58 females, aged between 30 and 75 years (median, 50 years). The following inclusion criteria were sued for the present study: i) Complete surgical R0 resection of the primary tumor; ii) pathologically confirmed diagnosis of gastric adenocarcinoma; iii) no chemotherapy or radiotherapy administered; and iv) absence of secondary malignancies. All patients provided a signed agreement for participation in the study and the protocol was approved by the Ethics Committees of The Third Affiliated Hospital of Nanjing University of Traditional Chinese Medicine and Wuxi XiShan People's Hospital. Written informed consent was obtained from all patients involved in the present study. The epidemiological, clinical and pathological features of patients included in the present study are summarized in Table I. The clinical outcome of the patients was followed for 1-60 months, from the date of surgery to either the date of mortality or August 30, 2015.

Immunohistochemistry and scoring. The tissues were fixed for $24 \mathrm{~h}$ in $4 \%$ paraformaldehyde and embedded in paraffin. The paraffin-embedded tissues were cut into $4 \mu \mathrm{m}$ sections. Then, tissue sections were deparaffinized in xylene and rehydrated through graded ethanol. Tissues were placed in $0.01 \mathrm{M}$ citrate buffer and incubated at $100^{\circ} \mathrm{C}$ for $20 \mathrm{~min}$ for antigen retrieval. Tissues were blocked with a $3 \%$ hydrogen peroxide solution to inhibit endogenous peroxidase activity and washed with PBS, Subsequently, sections were incubated with $5 \%$ normal rabbit serum (Abcam, Cambridge, UK) for $30 \mathrm{~min}$ at room temperature to block non-specific binding sites. The slides were subsequently incubated with a TAK1 antibody (1:50; catalog no. sc-7162; Santa Cruz Biotechnology, Inc., Dallas, TX, USA) at $4^{\circ} \mathrm{C}$ overnight, followed by incubation with a horseradish peroxidase (HRP)-conjugated secondary antibody (dilution 1:1,000; catalog no. ab6721; Abcam) for $60 \mathrm{~min}$ at room temperature. The sections were then developed in $0.05 \%$ diaminobenzidine and counterstained with $0.1 \%$ hematoxylin and eosin (H\&E) for $5 \mathrm{~min}$ at room temperature prior to dehydration and mounting. Evaluation of immunostaining in tumor cells was objectively performed by two pathologists under a light microscope at high magnification (x400). TAK1 staining was determined semi-quantitatively according to the intensity observed $(0=$ no staining; $1=$ weak staining; $2=$ moderate staining; and $3=$ strong staining) and the percentage of positive cells ( 0 , none or $<5 \% ; 1,5-20 \% ; 2,21-40 \%$; and $3,>40 \%)$. Scores of $0-2$ were considered to be negative expression and scores of 3-6 were considered to be positive expression. Cells were counted in at least three randomly selected fields (at x 400 magnification) in the tumor areas.

Cell culture. The MGC803 human gastric cancer cell line was purchased from Cell Bank of Type Culture Collection of Chinese Academy of Sciences (Shanghai, China). MGC803 cells were cultured at $37^{\circ} \mathrm{C}$ and $5 \% \mathrm{CO}_{2}$ and saturation humidity in RPMI-1640 medium (Gibco; Thermo Fisher Scientific, Inc.), with $10 \%$ fetal bovine serum, $100 \mathrm{U} / \mathrm{ml}$ penicillin (Thermo Fisher Scientific), and $100 \mathrm{mg} / \mathrm{ml}$ streptomycin (Thermo Fisher Scientific, Inc.). The cells adhered to the flask wall and grew into a single-cell monolayer and were passaged every 2-3 days. Cells in the exponential growth phase were harvested for subsequent experiments. There were four experimental groups: Control (without any intervention); vehicle treatment [1\% dimethylsulfoxide (DMSO)]; low-dose OZ (3 $\mu \mathrm{M})$; and high-dose OZ $(6 \mu \mathrm{M})$. TAK1 kinase inhibitor, OZ was purchased from Tocris Bioscience, Bristol, UK (catalog no. 3604).

MTT assay. Cells were plated in 96-well plates at a density of $5 \times 10^{3} /$ well and treated with $6 \mu \mathrm{M} \mathrm{OZ}$ or vehicle for 24,48 and $72 \mathrm{~h}$ at $37^{\circ} \mathrm{C}$. At the aforementioned time points, $20 \mu 1 \mathrm{MTT}$ substrate $(5 \mathrm{mg} / \mathrm{ml})$ was added to the cells and incubated at $37^{\circ} \mathrm{C}$ for $4 \mathrm{~h}$. The resulting colored product was made soluble in $200 \mu \mathrm{l}$ DMSO. Spectrometric absorbance at $490 \mathrm{~nm}$ was quantified using a microplate reader. Each cell line was established in quadruplicate wells and repeated three times.

Invasion assay. Cell invasion activity was determined using a BD BioCoat Matrigel Invasion Chamber (8- $\mu \mathrm{m}$; BD Biosciences, Franklin Lakes, NJ, USA). Briefly, gastric cancer cells were harvested and added to the upper chamber at a cell density of $2 \times 10^{5}$ cells $/ \mathrm{ml}$ in RPMI-1640 medium without FBS and treated with $6 \mu \mathrm{M} \mathrm{OZ}$ or DMSO. RPMI-1640 medium with $10 \%$ FBS was added to the lower chamber. The chambers were incubated for $48 \mathrm{~h}$ at $37^{\circ} \mathrm{C}$ and $5 \% \mathrm{CO}_{2}$. At the end of the incubation period, cells that had invaded through the membrane were subsequently fixed with $4 \%$ paraformaldehyde for $15 \mathrm{~min}$ and stained with $0.5 \%$ crystal violet for $30 \mathrm{~min}$ at room temperature. Cells were observed under $\mathrm{x} 40$ magnification with a ZEISS light microscope and counted. Each experiment was performed in triplicate and repeated three times.

Flow cytometry analysis. Annexin V-fluorescein isothiocyanate (FITC) apoptosis detection kit was used to analyze the apoptosis rate according to the manufacturer's protocol (Nanjing KeyGen Biotech Co., Ltd., Nanjing, China). MGC803 cells were seeded in six-well plates $\left(1 \times 10^{6}\right.$ cells/well) at $37^{\circ} \mathrm{C}$ and treated with $6 \mu \mathrm{M} \mathrm{OZ}$ or vehicle for 24,48 and $72 \mathrm{~h}$. Cells were dissociated using trypsin, then centrifuged at $400 \mathrm{xg}$ for 5 min. Next, cells were washed twice with PBS and centrifuged at $400 \mathrm{x}$ g for $5 \mathrm{~min}$. For apoptosis analysis, the cell pellet was resuspended in $500 \mu \mathrm{l}$ binding buffer. Then, $5 \mu \mathrm{l}$ Annexin V-FITC and $5 \mu \mathrm{l}$ propidiumiodide (PI) was added to the cell suspension, which was gently mixed and incubated at room temperature, and was protected from light, for $15 \mathrm{~min}$. 
Table I. Clinicopathological parameters and patients with positive expression of TAK1 in gastric cancer.

\begin{tabular}{|c|c|c|c|c|}
\hline \multirow[b]{2}{*}{ Clinicopathological parameters } & \multirow[b]{2}{*}{ Total } & \multicolumn{2}{|c|}{ TAK1 expression } & \multirow[b]{2}{*}{ P-value (Chi-square test) } \\
\hline & & Positive & Negative & \\
\hline Gender & & & & 0.96 \\
\hline Male & 81 & 57 & 24 & \\
\hline Female & 58 & 41 & 17 & \\
\hline Age, years & & & & 0.41 \\
\hline$<60$ & 62 & 42 & 20 & \\
\hline$\geq 60$ & 77 & 56 & 21 & \\
\hline Tumor size, cm & & & & 0.52 \\
\hline$<5$ & 60 & 44 & 16 & \\
\hline$\geq 5$ & 79 & 54 & 25 & \\
\hline Neural/vascular invasion & & & & 0.51 \\
\hline Yes & 42 & 28 & 14 & \\
\hline No & 97 & 70 & 27 & \\
\hline Tumor grade & & & & 0.26 \\
\hline I and II & 61 & 40 & 21 & \\
\hline III & 78 & 58 & 20 & \\
\hline T stage & & & & 0.60 \\
\hline $\mathrm{T} 1$ and $\mathrm{T} 2$ & 24 & 18 & 6 & \\
\hline $\mathrm{T} 3$ and $\mathrm{T} 4$ & 115 & 80 & 35 & \\
\hline $\mathrm{N}$ stage & & & & $<0.001$ \\
\hline No & 28 & 11 & 17 & \\
\hline N1-N3 & 111 & 87 & 24 & \\
\hline M stage & & & & 0.77 \\
\hline M0 & 131 & 92 & 39 & \\
\hline M1 & 8 & 6 & 2 & \\
\hline Pathological stage & & & & $<0.001$ \\
\hline I and II & 47 & 18 & 29 & \\
\hline III and IV & 92 & 80 & 12 & \\
\hline
\end{tabular}

TAK 1 , transforming growth factor- $\beta$-activated kinase 1 ; T, tumor; $\mathrm{N}$, node; $\mathrm{M}$, metastasis.

Within $1 \mathrm{~h}$, the cells were analyzed via flow cytometry using a BD FACSCanto II instrument (BD Biosciences, San Jose, CA, USA), and FlowJo software version 9.5.3 (Tree Star, Inc., Ashland, OR, USA).

Western blot analysis. MGC803 cells were treated with $6 \mu \mathrm{M}$ $\mathrm{OZ}$ for $48 \mathrm{~h}$ at $37^{\circ} \mathrm{C}$. Then, washed twice with PBS and centrifuged at $12,000 \mathrm{x}$ g for $15 \mathrm{~min}$ at $4^{\circ} \mathrm{C}$. A total cellular protein extraction kit (Beyotime Institute of Biotechnology, Haimen, China) was used to extract the total protein, and the nucleoprotein extraction kit (Beyotime Institute of Biotechnology) was used to extract nucleoprotein, according to the manufacturer's protocol. Isolation of mitochondrial and cytosolic proteins was performed using the Mitochondria/Cytosol Fractionation kit (Bi Yuntian Biological Technology Institution). Protein concentrations were determined using a BCA protein assay (Beyotime Institute of Biotechnology). Cell lysate was boiled for $12 \mathrm{~min}$, and samples ( $40 \mu \mathrm{g}$ protein per lane) were separated on $5-20 \%$ gradient SDS-PAGE gels. Proteins were subsequently transferred to polyvinylidene difluoride membranes (EMD Millipore, Billerica, MA, USA), which were blocked overnight in $5 \%$ non-fat milk at $4^{\circ} \mathrm{C}$. The membranes were incubated with the following primary antibodies at a dilution of 1:1,000: (p)-TAK1 (Thr187) (catalog no. 4536), pro-caspase 3 (catalog no. 9665), cyt $c$ (catalog no. 11940), cyclin D1 (catalog no. 2978), Bcl-2 apoptosis regulator (Bcl-2; catalog no. 2827), voltage-dependent anion channel (catalog no. 4661), cleaved caspase 3 (catalog no. 9654), matrix metallopeptidase (MMP) 9 (catalog no. 13667), p65 (catalog no. 4764), histone 3 (catalog no. 4499) and $\beta$-actin (catalog no. 8457) at $4^{\circ} \mathrm{C}$ overnight. All primary antibodies were obtained from Cell Signaling Technology, Inc. (Danvers, MA, USA). The membranes were then washed with $1 \mathrm{x}$ TBS containing $0.1 \%$ Tween-20, incubated with anti-rabbit IgG conjugated to HRP (dilution, 1:1,000; Cell Signaling Technology, Inc. catalog no. 7074) for $1 \mathrm{~h}$ at room temperature, and washed with 1xTBS containing $0.1 \%$ Tween-20 three times for $10 \mathrm{~min}$ each. Proteins were visualized using an Enhanced Chemiluminescence reagent 
(Pierce; Thermo Fisher Scientific, Inc., catalog no. 32106). The experiments were repeated at least 3 times. Densitometry analysis was performed using ImageJ software version 1.48 (National Institutes of Health).

Statistical analysis. Data are expressed as mean \pm standard deviation. SPSS version 19.0 (IBM, Armonk, NY, USA) was used for statistical analysis. Count data were analyzed with a $\chi^{2}$ test. Survival curves of the patients were compared using the Kaplan-Meier method and analyzed by the log-rank test. One-way analysis of variance followed by the Tukey post test was used to analyze differences between groups. $\mathrm{P}<0.05$ was considered to indicate a statistically significant difference.

\section{Results}

TAK1 protein expression in gastric cancer tissue and the association with clinical pathology. All gastric cancer tissue specimens and the adjacent normal tissue specimens used in the current study were verified using hematoxylin and eosin staining (Fig. 1). Immunohistochemistry revealed that the cytoplasm of gastric cancer cells appeared yellow or brown in a diffuse pattern, indicating high TAK1 expression; however, TAK1 expression was reduced in the adjacent normal tissues (Fig. 1).

To investigate the biological significance of TAK1 expression in gastric cancer, the patients were divided into two groups according to TAK1 immunostaining: the TAK1 negative group and the TRAF6 positive group. TAK1 positive expression was quantified as $70.5 \%$ in gastric cancer tissue samples and $25.9 \%$ in the adjacent normal tissues $(\mathrm{P}<0.001$; Table II). Furthermore, TAK1 expression was positively associated with advanced $\mathrm{N}$ stage and pathological stage, indicating that TAK1 protein expression level may be elevated during gastric cancer progression. No significant association was identified between TAK1 protein expression level and gender, age, tumor size, tumor grade, neural or vascular invasion, $\mathrm{T}$ stage or $\mathrm{M}$ stage $(\mathrm{P}>0.05$; Table $\mathrm{I})$. Kaplan-Meier survival analysis (Fig. 2) demonstrated that the median 5-year survival was 21 months in patients with positive TAK1 expression, which was significantly lower compared with patients with negative TAK1 expression (41 months; $\mathrm{P}=0.009)$.

Effect of OZ, the TAK1 inhibitor, on apoptosis. To evaluate the effects of OZ, the TAK1 inhibitor, on MGC803 cells, the present study examined the apoptotic properties of MGC803 cells incubated with OZ. The Annexin V and propidium iodide dual staining revealed that MGC803 cells from the TAK1 inhibitor treatment groups ( 3 and $6 \mu \mathrm{M}$ ) had a significantly greater percentage of apoptotic cells compared with the vehicle-treated group ( $\mathrm{P}<0.05$; Fig. 3A). To further investigate the cellular basis of the apoptotic response observed in the MGC803 cell line, the expression of apoptosis-associated proteins was investigated using western blotting. The aforementioned experiments confirmed that the high dose $\mathrm{OZ}$ $(6 \mu \mathrm{M})$ effectively promoted apoptosis in MGC803 cells, $6 \mu \mathrm{M}$ $\mathrm{OZ}$ was used for the subsequent experiments investigating the apoptotic mechanism. As demonstrated in Fig. 3B-D, OZ treatment significantly reduced the expression of mitochondrial cyt
Table II. Analysis of TAK1 expression level in gastric cancer tissues and adjacent normal tissues.

\begin{tabular}{lcrr}
\hline & \multicolumn{2}{c}{ TAK1 expression } & \\
\cline { 2 - 3 } Tissue & Positive (\%) & Negative (\%) & P-value \\
\hline Gastric cancer & $98(70.5)$ & $41(29.5)$ & $<0.001$ \\
Adjacent normal & $36(25.9)$ & $103(74.1)$ & \\
\hline
\end{tabular}

TAK1, transforming growth factor- $\beta$-activated kinase 1 .

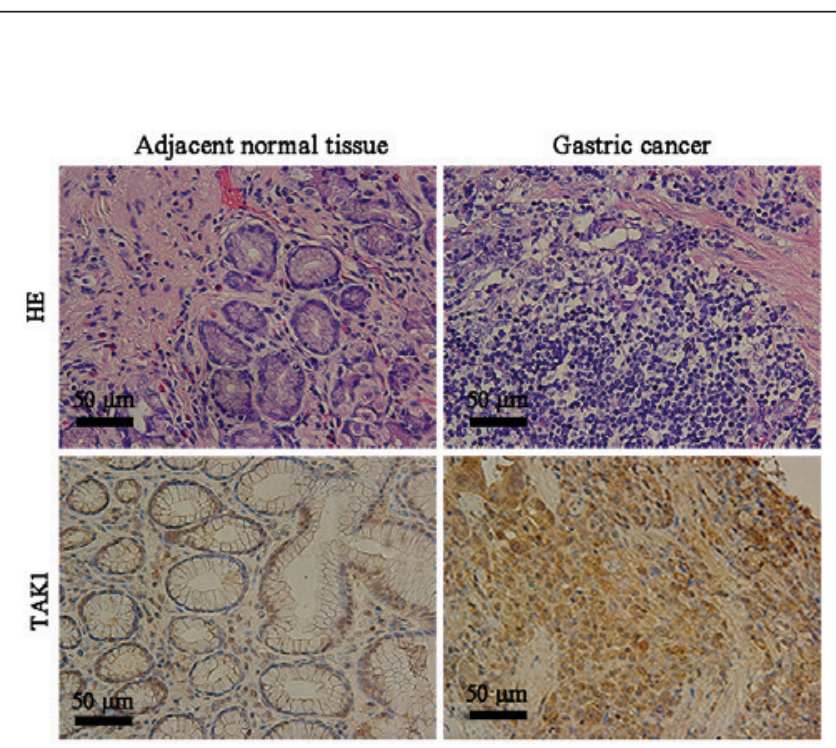

Figure 1. H\&E and immunohistochemical staining of gastric cancer tissues and adjacent normal tissues. Upper images present $\mathrm{H} \& \mathrm{E}$ staining results and lower images present results for TAK1 immunohistochemical staining. Scale bar, $50 \mu \mathrm{M}$. H\&E, hematoxylin and eosin; TAK1, transforming growth factor- $\beta$-activated kinase 1 .

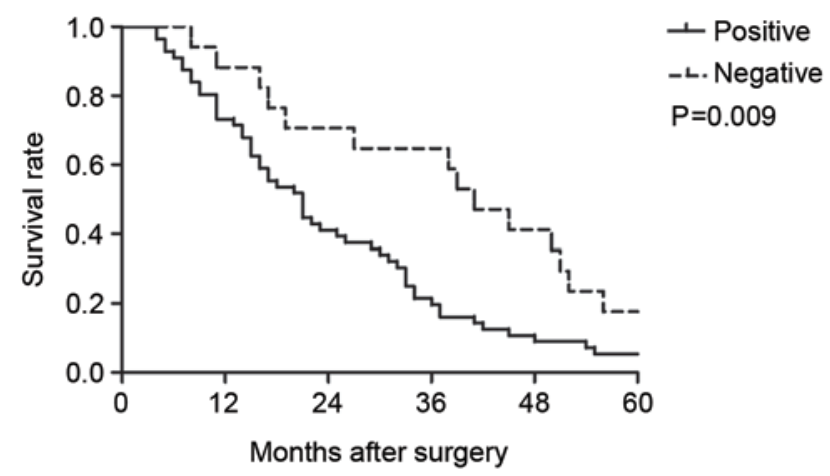

Figure 2. Overall survival of patients with gastric cancer and TAK1 expression level. Kaplan-Meier curves demonstrated that the 5-year survival rate was significantly higher in patients with negative TAK1 expression compared with patients with positive TAK1 expression. TAK1, transforming growth factor- $\beta$-activated kinase 1 .

$c(\mathrm{P}<0.05$; Fig. 3B), Bcl-2 ( $\mathrm{P}<0.05$; Fig. 3C) and procaspase 3 $(\mathrm{P}<0.05$; Fig. $3 \mathrm{C})$ compared with the vehicle treatment group. Conversely, cleaved caspase $3(\mathrm{P}<0.05$; Fig. $3 \mathrm{C})$ and cytosolic cyt $c(\mathrm{P}<0.05$; Fig. 3D) expression levels were significantly greater in the OZ-treated group compared with the vehicle group. 
A

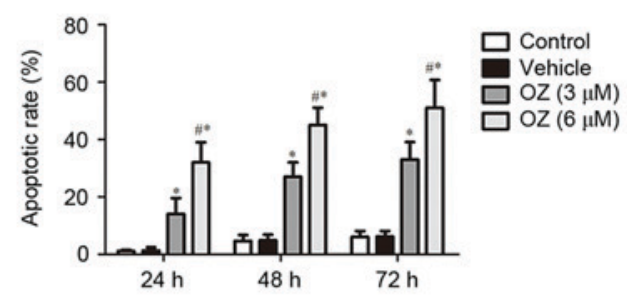

B

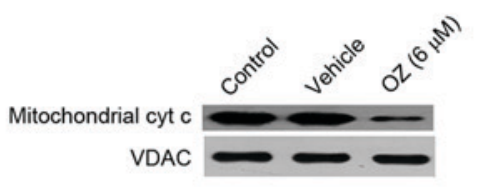

c

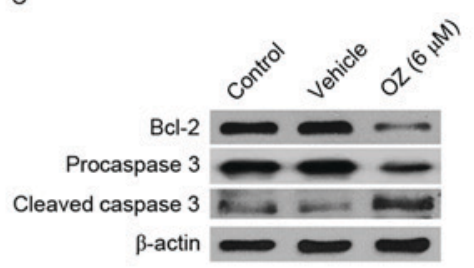

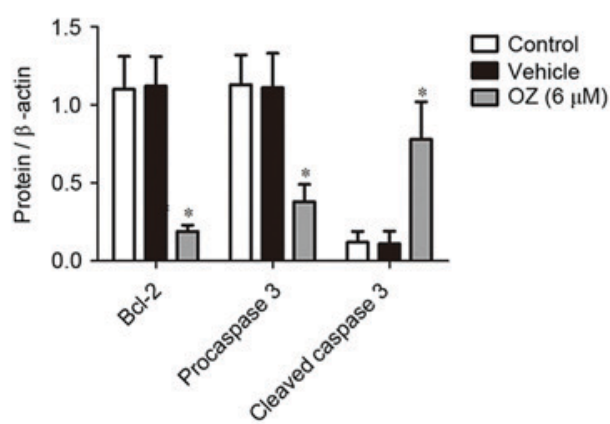

D

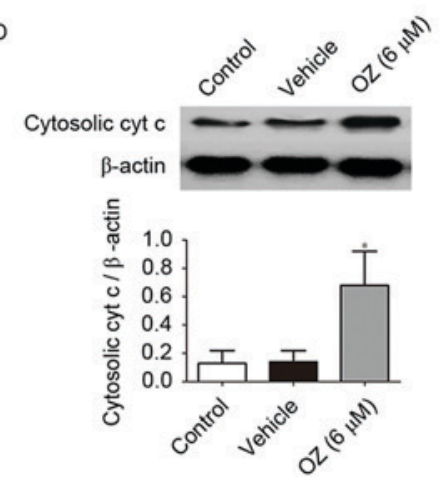

Figure 3. Effect of $\mathrm{OZ}$ on the apoptosis of gastric cancer cells. (A) OZ treatment ( 3 and $6 \mu \mathrm{M}$ ) significantly increased the apoptosis rate of MGC803 cells compared with the vehicle treatment group. The effect of $\mathrm{OZ}$ on the protein levels of (B) mitochondrial cyt $c$, (C) Bcl-2, procaspase 3 and cleaved caspase 3 , and (D) cytosolic cyt $c$ at $48 \mathrm{~h}$ after treatment were determined. ${ }^{*} \mathrm{P}<0.05$ vs. vehicle and ${ }^{\#} \mathrm{P}<0.05$ vs. $3 \mu \mathrm{M} \mathrm{OZ}$. OZ, 5Z-7-oxozeaenol; cyt $c$, cytochrome $c$; Bcl-2, Bcl-2 apoptosis regulator; VDAC, voltage-dependent anion channel.

A

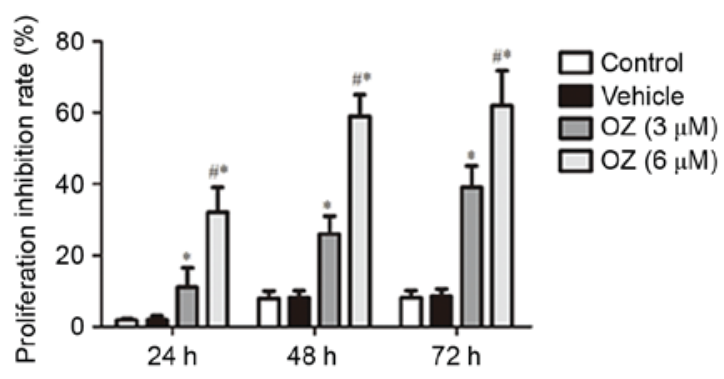

B
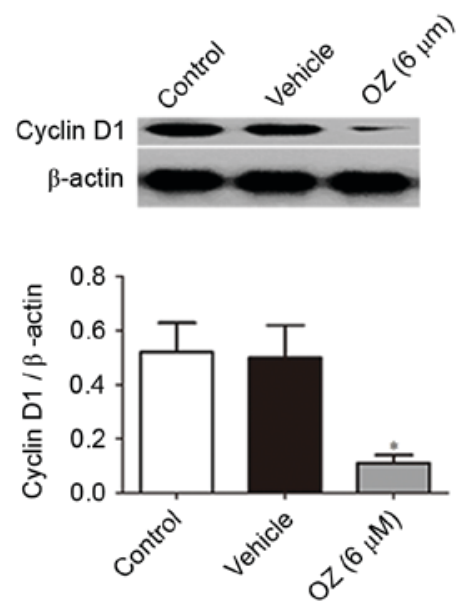

Figure 4. Effect of OZ on the proliferation of MGC803 human gastric cancer cells. (A) OZ (3 and $6 \mu \mathrm{M}$ ) significantly inhibited the proliferation of gastric cancer cells compared with the vehicle treatment group. (B) Effect of $\mathrm{OZ}(6 \mu \mathrm{M})$ on the protein expression of cyclin D1 at $48 \mathrm{~h}$ post-treatment was determined by western blot analysis. OZ significantly inhibited the protein expression levels of cyclin D1 compared with the vehicle treatment group. "P $<0.05$ vs. vehicle and ${ }^{\#} \mathrm{P}<0.05$ vs. $3 \mu \mathrm{M} \mathrm{OZ}$. OZ, 5Z-7-oxozeaenol.

Effect of OZ treatment on cell proliferation. To verify the effect of OZ treatment on tumor growth in MGC803 cells, cell proliferation was examined using an MTT assay. It was revealed that $\mathrm{OZ}$ treatment significantly inhibited the growth of MGC803 cells in a time-dependent manner ( $\mathrm{P}<0.05$; Fig. 4A) compared with vehicle-treated cells. As cyclin D1 has previously been reported to have an important role in gastric cancer proliferation $(15,16)$, the cyclin D1 protein expression level was examined by western blot analysis. The findings indicated that cyclin D1 expression was significantly downregulated in the $\mathrm{OZ}$ treatment group compared with the vehicle-treated group ( $\mathrm{P}<0.05$; Fig. 4B).

Effect of OZ treatment on cell invasion. The present study further investigated the effect of $\mathrm{OZ}$ treatment on the invasive behavior of MGC803 cells. As presented in Fig. 5A and B, 
A

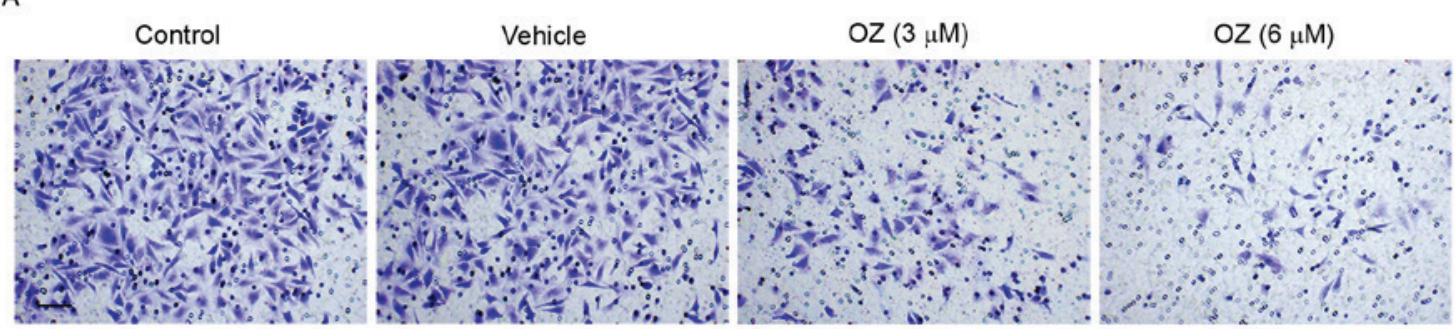

B

C
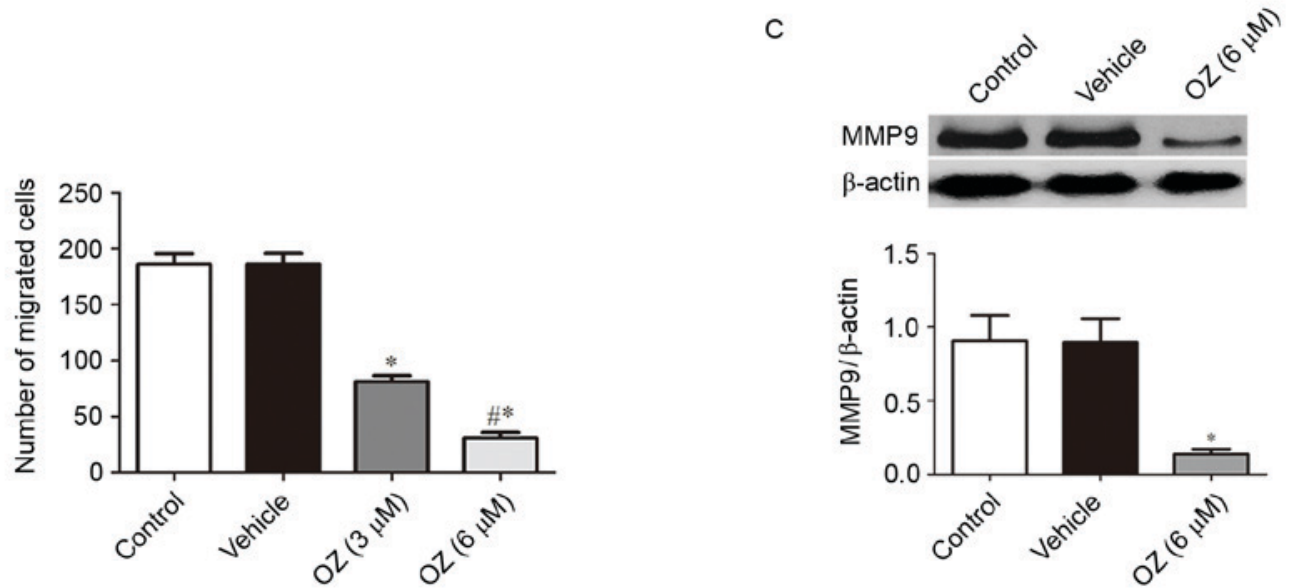

Figure 5. Effect of OZ on cell invasion ability of the MGC803 cell line. (A) Representative images and (B) quantification of invasive cells, as determined by the invasion assay. OZ (3 and $6 \mu \mathrm{M})$ significantly inhibited gastric cancer cell invasion compared with the vehicle treatment group. (C) Effect of OZ (6 $\mu \mathrm{M})$ on the protein expression of MMP9 at $48 \mathrm{~h}$ post-treatment was determined by western blot analysis. OZ treatment significantly inhibited the protein expression of MMP9 compared with the vehicle treatment group. ${ }^{*} \mathrm{P}<0.05$ vs. vehicle and ${ }^{\prime \prime} \mathrm{P}<0.05$ vs. $3 \mu \mathrm{M}$ OZ. OZ, 5Z-7-oxozeaenol; MMP9, matrix metallopeptidase-9.

A
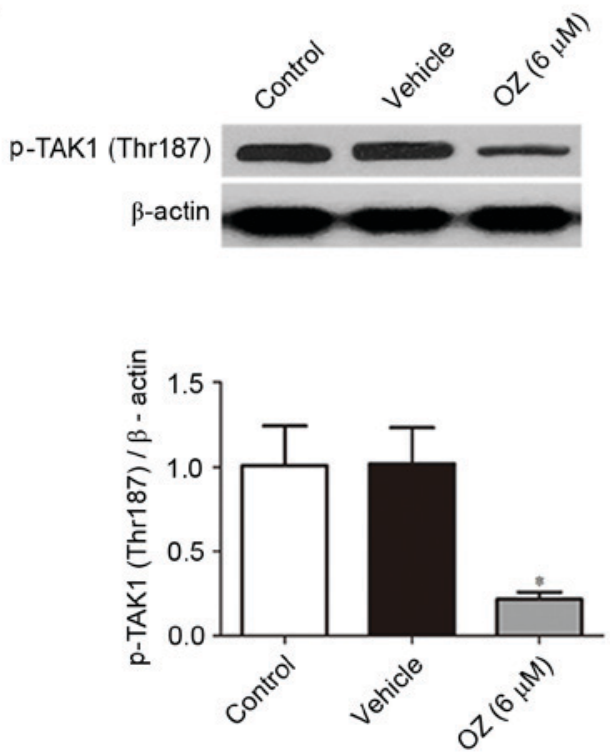

B
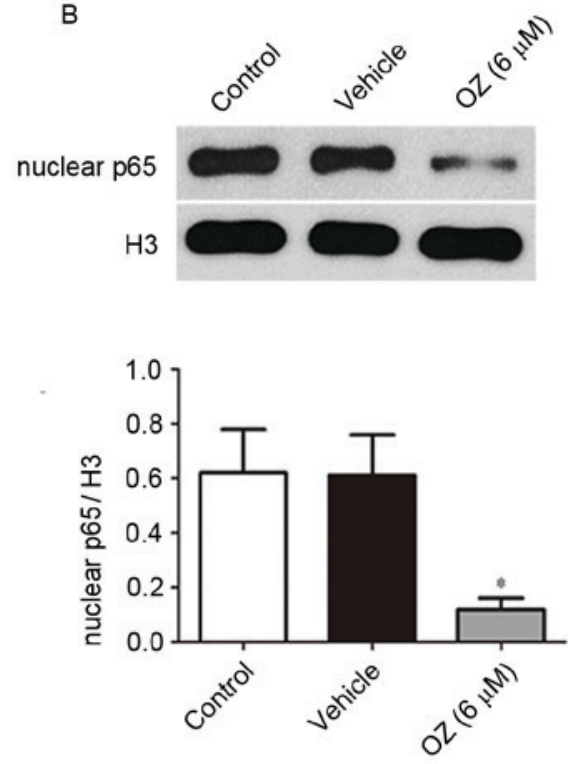

Figure 6. Effect of OZ on the protein expression of p-TAK1 (Thr187) and nuclear p65. OZ (6 $\mu \mathrm{M})$ treatment significantly inhibited the expression of (A) p-TAK1 (Thr187) and (B) nuclear $\mathrm{p} 65$ at $48 \mathrm{~h}$ post-treatment compared with the vehicle treatment group. " $\mathrm{P}<0.05$ vs. vehicle. OZ, 5Z-7-oxozeaenol; TAK1, transforming growth factor- $\beta$-activated kinase 1; p-TAK1, phosphorylated TAK1; Thr, threonine; H3, histone H3.

OZ treatment significantly reduced the invasive ability of MGC803 cells compared with the vehicle-treated group $(\mathrm{P}<0.05)$. As MMP9 has been previously reported to have an important role in gastric cancer invasion (17), the present study also investigated the expression level of MMP9 protein by western blot analysis and demonstrated that MMP9 expression was significantly downregulated in the $\mathrm{OZ}$ treatment group compared with the vehicle treatment group $(\mathrm{P}<0.05$; Fig. $5 \mathrm{C})$.

Effect of $O Z$ treatment on the TAK1/NF- $\kappa B$ signaling pathway. Following treatment with $\mathrm{OZ}$ for $48 \mathrm{~h}$, the expression levels of p-TAK1 (Thr187) and nuclear p65 protein were 
detected by western blot analysis. As presented in Fig. 6, OZ treatment significantly reduced p-TAK1 (Thr187) and nuclear p65 expression levels compared with the vehicle treatment group (both $\mathrm{P}<0.05$ ).

\section{Discussion}

TAK1 is a serine/threonine protein kinase, which belongs to the family of MAPK kinases. TAK1 is a key kinase in the signal pathway of toll-like receptors and the interleukin-1 receptor. Previous reports have demonstrated that the TAK1-mediated signal transduction pathway is a key regulator in signal transduction and the chain reaction of stress responses, inflammation immunity and the occurrence and development of tumors $(7,12)$. In addition, previous studies have demonstrated a high expression of TAK1 in a variety of tumor tissues such as thyroid cancer, non-small cell lung carcinoma and breast cancer, and an association with tumor occurrence, development and invasion $(8,9,18)$. TAK1 regulates the activation of the MAPK and NF- $\mathrm{BB}$ signaling pathways (7). Furthermore, abnormal activation of the MAPK signaling pathway is a hallmark of gastric cancer tissues and inhibition of MAPK activity may significantly inhibit the proliferation and invasion of gastric cancer cells, thus promoting apoptosis (19). In addition, the high expression of $\mathrm{NF}-\kappa \mathrm{B}$ in gastric cancer tissues was significantly associated with poor prognosis of patients with gastric cancer $(20,21)$. Inhibition of the NF- $\kappa \mathrm{B}$ signaling pathway may also inhibit the proliferation and invasion of gastric cancer cells, subsequently promoting apoptosis (22). Comprehensive analysis of previous research indicated that TAK1 may also have an important role in the occurrence and development of gastric cancer. The findings of the current study demonstrated that $25.9 \%$ of normal (non-neoplastic) gastric mucosae tissue samples exhibited positive TAK1 expression; therefore, it is possible that this regulation occurs at a transcriptional level as TAK1 has a key role in signal transduction in normal tissues (12). TAK1 protein expression was significantly increased in gastric cancer tissues compared with the normal tissues, which was consistent with the findings of a previous study (23). Furthermore, the findings of the present study also demonstrated that TAK1 expression was associated with the advanced $\mathrm{N}$ stage and the pathological stage of gastric carcinoma. However, no significant association was identified in terms of gender, age, tumor size, tumor grade, neural or vascular invasion, $\mathrm{T}$ and $\mathrm{M}$ stage. In addition, the 5-year survival rate of patients with positive TAK1 expression was significantly lower compared with patients with negative TAK1 expression. Therefore, postoperative detection of TAK1 in gastric cancer tumor specimens may be used for a prognosis of the patient.

OZ is a selective inhibitor of TAK1 (13). Several recent studies demonstrated that $\mathrm{OZ}$ inhibited the proliferation and invasion of a variety of tumor cells, and promoted apoptosis $(8,24-26)$. Therefore, after confirming high expression of TAK1 in gastric cancer tissues, the present study further investigated the effects of TAK1 on the invasion and apoptosis of gastric cancer cells, and the potential underlying mechanisms using an in vitro culture of MGC803 human gastric carcinoma cells. The present study used a previously reported dose of OZ $(8,24,26,27)$, and the findings indicated that OZ treatment significantly inhibited the invasion and proliferation of gastric cancer cells, whilst promoting apoptosis. A previous report demonstrated that the phosphorylation of threonine 187 at the loci of TAK1 protein kinase was important for the activation of this kinase (28). Previous studies have demonstrated that $\mathrm{OZ}$ inhibited the expression of p-TAK1 (Thr187) (29) and significantly reduced the expression of p65 in the nucleus $(30,31)$. Previous studies have demonstrated that cleaved caspase 3 has an important role in the apoptosis of gastric cancer cells. High protein expression level of cleaved caspase 3 significantly promoted the apoptosis of gastric cancer cells, whereas downregulation of $\mathrm{Bcl}-2$ promoted cyt $c$ release and induced the apoptosis of gastric cancer cells (32-35). Importantly, cleaved caspase 3 and $\mathrm{Bcl}-2$ were both regulated by $\mathrm{NF}-\kappa \mathrm{B}$ signaling pathways (36-38). A previous study also demonstrated that inhibition of TAK1 was associated with the release of cyt $c$ from the mitochondria, which served as an important initial step for apoptosis (39). The findings of the present study indicated that $\mathrm{OZ}$ treatment significantly increased cleaved caspase-3 and cytosolic cyt $c$ expression and inhibited the expression of Bcl-2 in gastric cancer cells. These findings may elucidated the underlying mechanism whereby OZ functions as a tumor suppressor by inducing apoptosis. In addition, previous reports indicated that MMP9, which is regulated by $\mathrm{NF}-\kappa \mathrm{B}(40)$, had an important role in the invasion of gastric cancer cells $(17,41)$. The current study demonstrated that $\mathrm{OZ}$ significantly inhibited the expression of MMP9, which may be one of the molecular mechanisms by which $\mathrm{OZ}$ inhibited the invasion of gastric cancer cells. NF- $\kappa \mathrm{B}$ has also been reported to stimulate the transcription of cyclin D1 (42), which is a key regulator of gastric cancer proliferation $(15,16)$. In the current study, OZ treatment significantly reduced the cyclin D1 expression level. This may be a potential method by which OZ inhibited gastric cancer cell proliferation.

As only one gastric cancer cell line was utilized for mechanistic studies in the current study, the results may be limited and a variety of gastric cancer cell lines are required for future investigation of the relevant mechanisms. Previous studies have revealed that TAK1 may have a biphasic role in tumorigenesis and promote tumor growth during the early development of a tumor and delay metastasis in advanced tumor stages $(43,44)$. Lam et al (43) hypothesized that this discrepancy may be due the influence of other surrounding cell types, such as cancer-associated fibroblasts. Based on these observations, the role of TAK1 in gastric cancer development may require further investigation using in vivo experiments.

In conclusion, the present study demonstrated that TAK1 expression was elevated in gastric carcinoma tissues and was associated with the poor prognosis of patients with gastric cancer. OZ, the specific inhibitor of TAK1, significantly inhibited the proliferation and invasion of gastric cancer cells and promoted cell apoptosis, indicating that TAK1 may be a novel target for the treatment of gastric cancer and that $\mathrm{OZ}$ may have the potential to be developed as a novel drug for the treatment of gastric cancer.

\section{Acknowledgements}

The present study was supported by the National Natural Science Foundation (grant no. 81470866). 


\section{References}

1. Digklia A and Wagner AD: Advanced gastric cancer: Current treatment landscape and future perspectives. World J Gastroenterol 22: 2403-2414, 2016.

2. Lordick F and Janjigian YY: Clinical impact of tumour biology in the management of gastroesophageal cancer. Nat Rev Clin Oncol 13: 348-360, 2016.

3. Tomasello G, Ghidini M, Liguigli W, Ratti M, Toppo L and Passalacqua R: Targeted therapies in gastric cancer treatment: Where we are and where we are going. Invest New Drugs 34: 378-393, 2016.

4. Karimi P, Islami F, Anandasabapathy S, Freedman ND and Kamangar F: Gastric cancer: Descriptive epidemiology, risk factors, screening and prevention. Cancer Epidemiol Biomarkers Prev 23: 700-713, 2014.

5. Liu X and Meltzer SJ: Gastric cancer in the era of precision medicine. Cell Mol Gastroenterol Hepatol 3: 348-358, 2017.

6. Lee SY and Oh SC: Changing strategies for target therapy in gastric cancer. World J Gastroenterol 22: 1179-1189, 2016.

7. Sakurai H: Targeting of TAK1 in inflammatory disorders and cancer. Trends Pharmacol Sci 33: 522-530, 2012.

8. Huang HL, Chiang $\mathrm{CH}$, Hung WC and Hou MF: Targeting of TGF- $\beta$-activated protein kinase 1 inhibits chemokine (C-C motif) receptor 7 expression, tumor growth and metastasis in breast cancer. Oncotarget 6: 995-1007, 2015.

9. Lin P, Niu W, Peng C, Zhang Z and Niu J: The role of TAK1 expression in thyroid cancer. Int J Clin Exp Pathol 8: 14449-14456, 2015.

10. Wu M, Shi L, Cimic A, Romero L, Sui G, Lees CJ, Cline JM, Seals DF, Sirintrapun JS, McCoy TP, et al: Suppression of Tak1 promotes prostate tumorigenesis. Cancer Res 72: 2833-2843, 2012.

11. Bosman MC, Schepers H, Jaques J, Brouwers-Vos AZ, Quax WJ, Schuringa JJ and Vellenga E: The TAK1-NF- $\kappa$ B axis as therapeutic target for AML. Blood 124: 3130-3140, 2014.

12. Kilty I and Jones LH: TAK1 selective inhibition: State of the art and future opportunities. Future Med Chem 7: 23-33, 2015.

13. Wu J, Powell F, Larsen NA, Lai Z, Byth KF, Read J, Gu RF, Roth M, Toader D, Saeh JC and Chen H: Mechanism and in vitro pharmacology of TAK1 inhibition by (5Z)-7-Oxozeaenol. ACS Chem Biol 8: 643-650, 2013.

14. Fakhouri L, El-Elimat T, Hurst DP, Reggio PH, Pearce CJ, Oberlies NH and Croatt MP: Isolation, semisynthesis, covalent docking and transforming growth factor beta-activated kinase 1 (TAK1)-inhibitory activities of (5Z)-7-oxozeaenol analogues. Bioorg Med Chem 23: 6993-6999, 2015.

15. Arici DS, Tuncer E, Ozer H, Simek G and Koyuncu A: Expression of retinoblastoma and cyclin D1 in gastric carcinoma. Neoplasma 56: 63-67, 2009.

16. Seo JH, Jeong ES and Choi YK: Therapeutic effects of lentivirus-mediated shRNA targeting of cyclin D1 in human gastric cancer. BMC Cancer 14: 175, 2014.

17. Akter H, Park M, Kwon OS, Song EJ, Park WS and Kang MJ: Activation of matrix metalloproteinase-9 (MMP-9) by neurotensin promotes cell invasion and migration through ERK pathway in gastric cancer. Tumour Biol 36: 6053-6062, 2015.

18. Zhu J, Li Q, He JT and Liu GY: Expression of TAK1/TAB1 expression in non-small cell lung carcinoma and adjacent normal tissues and their clinical significance. Int J Clin Exp Pathol 8: 15801-15807, 2015.

19. Yang $M$ and Huang CZ: Mitogen-activated protein kinase signaling pathway and invasion and metastasis of gastric cancer. World J Gastroenterol 21: 11673-11679, 2015.

20. Li X, Tu J, Zhang D, Xu Z, Yang G, Gong L and Yu M: The clinical significance of HER-2 and NF-KB expression in gastric cancer. Hepatogastroenterology 60: 1519-1523, 2013.

21. Li Q, Yu YY, Zhu ZG, Ji YB, Zhang Y, Liu BY, Chen XH and Lin YZ: Effect of NF-kappaB constitutive activation on proliferation and apoptosis of gastric cancer cell lines. Eur Surg Res 37: 105-110, 2005.

22. Uetsuka H, Haisa M, Kimura M, Gunduz M, Kaneda Y, Ohkawa T, Takaoka M, Murata T, Nobuhisa T, Yamatsuji T, et al: Inhibition of inducible NF-kappaB activity reduces chemoresistance to 5-fluorouracil in human stomach cancer cell line. Exp Cell Res 289: 27-35, 2003.

23. Pak KH, Kim DH, Kim H, Lee DH and Cheong JH: Differences in TGF-b1 signaling and clinicopathologic characteristics of histologic subtypes of gastric cancer. BMC Cancer 16: 60, 2015 .
24. Zhang J, Li B, Wu H, Ou J, Wei R, Liu J, Cai W, Liu X, Zhao S, Yang J, et al: Synergistic action of 5Z-7-oxozeaenol and bortezomib in inducing apoptosis of Burkitt lymphoma cell line Daudi. Tumour Biol 37: 531-539, 2016.

25. Hrabe JE, O'Leary BR, Fath MA, Rodman SN, Button AM, Domann FE, Spitz DR and Mezhir JJ: Disruption of thioredoxin metabolism enhances the toxicity of transforming growth factor $\beta$-activated kinase 1 (TAK1) inhibition in KRAS-mutated colon cancer cells. Redox Biol 5: 319-327, 2015.

26. Cai PC, Shi L, Liu VW, Tang HW, Liu IJ, Leung TH, Chan KK, Yam JW, Yao KM, Ngan HY and Chan DW: Elevated TAK1 augments tumor growth and metastatic capacities of ovarian cancer cells through activation of NF- $\kappa B$ signaling. Oncotarget 5: 7549-7562, 2014

27. Fan Y, Cheng J, Vasudevan SA, Patel RH, Liang L, Xu X, Zhao Y, Jia W, Lu F, Zhang $\mathrm{H}$, et al: TAK1 inhibitor 5Z-7-oxozeaenol sensitizes neuroblastoma to chemotherapy. Apoptosis 18: 1224-1234, 2013.

28. Singhirunnusorn P, Suzuki S, Kawasaki N, Saiki I and Sakurai H: Critical roles of threonine 187 phosphorylation in cellular stress-induced rapid and transient activation of transforming growth factor-beta-activated kinase 1 (TAK1) in a signaling complex containing TAK1-binding protein TAB1 and TAB2. J Biol Chem 280: 7359-7368, 2005.

29. Choo MK, Kawasaki N, Singhirunnusorn P, Koizumi K, Sato S, Akira S, Saiki I and Sakurai H: Blockade of transforming growth factor-beta-activated kinase 1 activity enhances TRAIL-induced apoptosis through activation of a caspase cascade. Mol Cancer Ther 5: 2970-2976, 2006.

30. Cao H, Lu J, Du J, Xia F, Wei S, Liu X, Liu T, Liu Y and Xiang M: TAK1 inhibition prevents the development of autoimmune diabetes in NOD mice. Sci Rep 5: 14593, 2015.

31. Song Z, Zhu X, Jin R, Wang C, Yan J, Zheng Q, Nanda A, GrangerDN and Li G: Roles of the kinase TAK1 in CD 40-mediated effects on vascular oxidative stress and neointima formation after vascular injury. PloS One 9: e101671, 2014.

32. Guo JQ, Li SJ and Guo GX: Long noncoding RNA AFAP1-AS1 promotes cell proliferation and apoptosis of gastric cancer cells via PTEN/p-AKT pathway. Dig Dis Sci: Apr 27, 2017 (Epub ahead of print)

33. Tong K, Xin C and Chen W: Isoimperatorin induces apoptosis of the SGC-7901 human gastric cancer cell line via the mitochondria-mediated pathway. Oncol Lett 13: 518-524, 2017.

34. Wang D, Li Y, Cui P, Zhao Q, Tan BB, Zhang ZD, Liu Y and Jia N: Zerumbone induces gastric cancer cells apoptosis: Involving cyclophilin A. Biomed Pharmacother 83: 740-745, 2016.

35. Shen X, Si Y, Wang Z, Wang J, Guo Y and Zhang X: Quercetin inhibits the growth of human gastric cancer stem cells by inducing mitochondrial-dependent apoptosis through the inhibition of PI3K/Akt signaling. Int J Mol Med 38: 619-626, 2016.

36. Yang LQ, Fang DC, Wang RQ and Yang SM: Effect of NF-kappaB, survivin, Bcl-2 and Caspase 3 on apoptosis of gastric cancer cells induced by tumor necrosis factor related apoptosis inducing ligand. World J Gastroenterol 10: 22-25, 2004.

37. Chang MS, Lee HS, Jung EJ, Kim CW, Lee BL and Kim WH: Cell-cycle regulators, bcl-2 and NF-kappaB in Epstein-Barr virus-positive gastric carcinomas. Int J Oncol 27: 1265-1272, 2005.

38. Dolcet X, Llobet D, Pallares J and Matias-Guiu X: NF-kB in development and progression of human cancer. Virchows Arch 446: 475-482, 2005.

39. Buglio D, Palakurthi S, Byth K, Vega F, Toader D, Saeh J, Neelapu SS and Younes A: Essential role of TAK1 in regulating mantle cell lymphoma survival. Blood 120: 347-355, 2012.

40. Park BB, Yoon Js, Kim Es, Choi J, Won Yw, Choi Jh and Lee YY: Inhibitory effects of eupatilin on tumor invasion of human gastric cancer MKN-1 cells. Tumour Biol 34: 875-885, 2013.

41. Zhang QW, Liu L, Chen R, Wei YQ, Li P, Shi HS and Zhao YW: Matrix metalloproteinase-9 as a prognostic factor in gastric cancer: A meta-analysis. Asian Pac J Cancer Prev 13: 2903-2908, 2012.

42. Hinz M, Krappmann D, Eichten A, Heder A, Scheidereit C and Strauss M: NF-kappaB function in growth control: Regulation of cyclin D1 expression and G0/G1-to-S-phase transition. Mol Cell Biol 19: 2690-2698, 1999.

43. Lam CR, Tan C, Teo Z, Tay CY, Phua T, Wu YL, Cai PQ, Tan LP, Chen X, Zhu P and Tan NS: Loss of TAK1 increases cell traction force in a ROS-dependent manner to drive epithelial-mesenchymal transition of cancer cells. Cell Death Dis 4: e848, 2013.

44. Omori E, Matsumoto K, Zhu S, Smart RC and Ninomiya-Tsuji J: Ablation of TAK1 upregulates reactive oxygen species and selectively kills tumor cells. Cancer Res 70: 8417-8425, 2010. 